\title{
A Family of Novel Graph Kernels for Structural Pattern Recognition
}

\author{
Horst Bunke and Kaspar Riesen \\ Institute of Computer Science and Applied Mathematics, University of Bern, \\ Neubrückstrasse 10, CH-3012 Bern, Switzerland \\ \{bunke, riesen\}@iam. unibe.ch
}

\begin{abstract}
Recently, an emerging trend of representing objects by graphs can be observed. As a matter of fact, graphs offer a versatile alternative to feature vectors in pattern recognition, machine learning and data mining. However, the space of graphs contains almost no mathematical structure, and consequently, there is a lack of suitable methods for graph classification. Graph kernels, a novel class of algorithms for pattern analysis, offer an elegant solution to this problem. Graph kernels aim at bridging the gap between statistical and symbolic object representations. In the present paper we propose a general approach to transforming graphs into $n$-dimensional real vector spaces by means of graph edit distance. As a matter of fact, this approach results in a novel family of graph kernels making a wide range of kernel machines applicable for graphs. With several experimental results we prove the robustness and flexibility of our new method and show that our approach outperforms a standard graph classification method on several graph data sets of diverse nature.
\end{abstract}

\section{Introduction}

The field of pattern recognition can be divided into two sub-fields, namely the statistical and the structural approach. In statistical pattern recognition, patterns are represented by feature vectors $\left(x_{1}, \ldots, x_{n}\right) \in \mathbb{R}^{n}$. The recognition process is based on the assumption that patterns of the same class are located in a compact region of $\mathbb{R}^{n}$. In recent years a huge amount of methods for the classification of patterns represented by feature vectors have been proposed, such as Bayes classifier, neural network, support vector machine, and many more. Object representations given in terms of feature vectors have a number of useful properties 1]. For example, object similarity, or distance, can easily be computed by means of Euclidean distance. Computing the sum or weighted sum of two objects represented by vectors is straightforward, too. Yet graph-based representations, which are used in the field of structural pattern recognition, have a number of advantages over feature vectors. Graphs are much more powerful and flexible than vectors, as feature vectors provide no direct possibility to describe structural relations in the patterns under consideration. Furthermore, while the size of a graph can be adjusted to the size and complexity of the underlying pattern, vectors are constrained to a predefined length, which has to be preserved

L. Rueda, D. Mery, and J. Kittler (Eds.): CIARP 2007, LNCS 4756, pp. 20-31 2007.

(C) Springer-Verlag Berlin Heidelberg 2007 
for all patterns encountered in a particular application. On the other hand, a major drawback of graph representations is their lack of suitable methods for classification. This is mainly due to the fact that some of the basic operations needed in classification are not available for graphs.

It turns out that the lack of operations needed for the implementation of classification algorithms in the graph domain can be overcome by means of kernel methods 23]. Although some mathematical foundations of kernel methods have been developed long ago 4, the practical usefulness of kernel methods for the fields of pattern recognition, machine learning and data mining has been recognised only recently [5]. During the past ten years kernel methods have become one of the most rapidly emerging sub-fields in intelligent information processing. The vast majority of work in kernel methods is concerned with transforming a given feature space into a higher-dimensional space without computing the transformation explicitly for each individual feature vector. However, more recently the existence of kernels for symbolic data structures, especially for graphs, has been shown [6]. By means of suitable kernel functions, graphs can be mapped into vector spaces in an explicit or implicit fashion. Hence, rather than computing the Euclidean distance, a weighted sum, the average of a set of graphs, and similar quantities in the domain of graphs - which is actually not possible in general -, the corresponding operations are carried out in the target space of the kernel function, i.e. in a vector space. Consequently, a large class of classification algorithms, originally developed for feature vectors, become applicable to graphs. Hence, by means of kernel functions one can benefit from both the high representational power of graphs and the large repository of algorithmic tools available for feature vector representations of objects.

In the rest of this paper, we give a general introduction to graph kernels in Section 2. Then, in Section 3, a new family of graph kernels developed recently is introduced 789. In Section 4, an experimental evaluation of these kernels is described. Finally, conclusions are drawn in Section 5.

\section{Graph Kernels}

For a general introduction to kernel methods we refer to [2]. While the bulk of work on kernel methods has been concerned with mapping vectors from a lowto a high-dimensional space, recently also kernels on symbolic data structures have been proposed. In this section we give a brief survey on graph kernels. For an earlier survey see [6].

A well-known class of graph kernels is based on the analysis of random walks in graphs. These kernels measure the similarity of two graphs by the number of random walks in both graphs that have all or some labels in common [1011]. In [10] an important result is reported. It is shown that the number of matching walks in two graphs can be computed by means of the product graph of two graphs, without the need to explicitly enumerate the walks. In order to handle continuous labels the random walk kernel has been extended in [11. This extension allows one to also take non-identically labelled walks into account. The 
problem of tottering, is addressed in [12]. Tottering is the phenomenon that, in a walk, a node may be revisited immediately after it has been left. In order to prevent tottering, the random walk transition probability model is appropriately modified in 12 .

Another class of graph kernels is given by diffusion kernels (e.g. in [13]). The kernels of this class are defined with respect to a base similarity measure which is used to construct a valid kernel matrix. This base similarity measure only needs to satisfy the condition of symmetry and can be defined for any kind of objects.

An important contribution to graph kernels is the work on convolution kernels, which provides a general framework for dealing with complex objects that consist of simpler parts 14. Convolution kernels infer the similarity of complex objects from the similarity of their parts. The ANOVA kernel [15], for instance, is a particular convolution kernel, which uses a subset of the components of a composite object for comparison.

A number of additional kernels are discussed in [16 17/18]. In [16] support vector machines are applied to attributed graphs where the kernel matrices are based on approximations of the Schur-Hadamard inner product. The idea underlying the kernels in [17/18 is to find identical substructures in two graphs, such as common subgraphs, subtrees, and cycles.

In a recent $\mathrm{PhD}$ thesis [19] graph kernels derived from graph edit distance are introduced. These kernels can cope with any type of graph and are applicable to a wide spectrum of different applications.

\section{Novel Graph Kernels Using Vector Space Embedding Based on Edit Distance}

The present paper introduces a new class of graph kernels which are based on vector space embeddings of graphs by means of prototype selection and graph edit distance computation. In contrast to some other kernel methods, the approach proposed in this paper results in an explicit embedding of the considered graphs in a vector space. Hence, not only scalar products, but individual graph maps are available in the target space. We observe that this approach is more powerful than some other graph kernels for a number of reasons. First, as the map of each graph in the target vector space is explicitly computed, not only kernel machines, but also other non-kernelizable algorithms can be applied to the resulting vector representation. Secondly, there are almost no restrictions on the type of graphs the proposed method can deal with. It can be applied to directed or undirected graphs, and to graphs without or with labels on their nodes and/or edges. In case there are labels on the nodes and/or edges, these labels can be of any nature, for example, they can be elements from a finite or infinite set of discrete symbols, the set of integer numbers, real numbers, or real vectors. Thirdly, the method is versatile, i.e. it is possible to integrate domain specific knowledge about object similarity when defining the costs of the elementary edit operations. Hence the kernel can be made more discriminative by tuning graph similarity to the specific application area. 


\subsection{Graph Edit Distance}

Similarly to [19, the new graph kernels proposed in this paper make use of the edit distance of graphs. Therefore, we give a brief introduction to this graph distance measure in this section.

Graph edit distance is one of the most flexible graph distance measures that is available [20 21]. The key idea of graph edit distance is to define the dissimilarity, or distance, of graphs by the amount of distortion that is needed to transform one graph into another. These distortions are given by insertions, deletions, and substitutions of nodes and edges. Given two graphs - the source graph $g_{1}$ and the target graph $g_{2}$ - the idea is to delete some nodes and edges from $g_{1}$, relabel some of the remaining nodes and edges (substitutions) and possibly insert some nodes and edges, such that $g_{1}$ is finally transformed into $g_{2}$. A sequence of edit operations that transform $g_{1}$ into $g_{2}$ is called an edit path between $g_{1}$ and $g_{2}$. One can introduce cost functions for each edit operation measuring the strength of the given distortion. The idea of such cost functions is that one can define whether an edit operation represents a strong modification of the graph or not. Hence, between two structurally similar graphs, there exists an inexpensive edit path, representing low cost operations, while for structurally different graphs an edit path with high costs is needed. Consequently, the edit distance of two graphs is defined by the minimum cost edit path between two graphs. The edit distance can be computed by a tree search algorithm [20], or by a faster, suboptimal method 2223.

\subsection{Basic Embedding Approach}

The novel graph kernels described in this paper are based on the idea of embedding a population of graphs in an $m$-dimensional real vector space. Such an embedding is motivated by the lack of suitable classification algorithms in the graph domain. Another approach to graph embedding has been proposed in 24]. This method is based on algebraic graph theory and utilizes spectral matrix decomposition. Applying an error-tolerant string matching algorithm to the eigensystem of graphs to infer distances of graphs is proposed in [25]. These distances are then used to embed the graphs into a vector space by multidimensional scaling. In 26, features derived from the eigendecompostion of graphs are studied. In fact, such feature extraction defines an embedding of graphs into vector spaces, too. In our approach we will explicitly make use of graph edit distance. Hence, we can easily deal with various kinds of graphs (labelled, unlabelled, directed, undirected, etc.) and utilize domains specific knowledge in defining the dissimilarity of nodes and edges through edit costs. Thus a high degree of robustness against various graph distortions can be achieved. The idea underlying our method was originally developed for the problem of embedding sets of feature vectors in a dissimilarity space [27. In this paper we introduce a new version of this method for the domain of graphs.

Assume we have a labelled set of training graphs, $T=\left\{g_{1}, \ldots, g_{n}\right\}$. Let $d\left(g_{i}, g_{j}\right)$ be the edit distance between graph $g_{i}$ and $g_{j}$. After having selected 
a set $P=\left\{p_{1}, \ldots, p_{m}\right\}$ of $m \leq n$ prototypes from $T$, we compute the edit distance of a graph $g \in T$ to each prototype $p \in P$. This leads to $m$ dissimilarities, $d_{1}=d\left(g, p_{1}\right), \ldots, d_{m}=d\left(g, p_{m}\right)$, which can be interpreted as an $m$-dimensional vector $\left(d_{1}, \ldots, d_{m}\right)$. In this way we can transform any graph from the training set, as well as any other graph from a validation or testing set, into a vector of real numbers. Formally, if $T=\left\{g_{1}, \ldots, g_{n}\right\}$ is a training set of graphs and $P=\left\{p_{1}, \ldots, p_{m}\right\} \subseteq T$ is a set of prototypes, the mapping $t_{m}^{P}: T \rightarrow \mathbb{R}^{m}$ is defined as a function $t_{m}^{P}(g) \mapsto\left(d\left(g, p_{1}\right), \ldots, d\left(g, p_{m}\right)\right)$ where $d\left(g, p_{i}\right)$ is the graph edit distance between the graph $g$ and the $i$-th prototype.

\subsection{Prototype Selection}

The method described in Section 3.2 crucially depends on the prototypes. Therefore, an important problem to be solved is an appropriate choice of the prototype set $P=\left\{p_{1}, \ldots, p_{m}\right\}$. A good selection of $m$ prototypes seems to be crucial to succeed with the classification algorithm in the feature vector space. The prototypes should avoid redundancies in terms of selection of similar graphs, and prototypes should include as much information as possible. In this section we discuss five different algorithms for the task of prototype selection ${ }^{1}$. Note that all of the proposed prototype selection methods can be applied class-wise and class-independent, i.e. the selection can be executed over the whole training set, or the selection can be performed individually for each of the classes.

Centers. The CEnTERs prototype selector selects prototypes situated in the center of the graph set $T$. This is achieved by iteratively taking the set median graph out of the set $T$. The set median graph is the graph whose sum of distances to all other graphs in this set is minimal.

RANDOM. A random selection of $m$ prototypes from $T$ is performed.

Spanning. A set of prototypes, $P$, is selected by the Spanning prototype selector by means of the following iterative procedure. The first prototype selected is the set median graph. Each additional prototype selected by the spanning prototype selector is the graph the furthest away from the already selected prototype graphs.

$k$-Centers. The $k$-Centers prototype selector tries to adapt to the graph distrubution of set $T$ and selects graphs that are in the center of densely populated areas. First a $k$-means clustering procedure is applied to set $T$. The number of clusters to be found is equal to the number of prototypes to be selected. Once the clusters have been established, the median of each cluster is selected as a prototype.

TARGetsphere. The TARGETsPhere prototype selector first selects a graph $g_{c}$ situated in the center of $T$. After finding the center graph, the graph $g_{f} \in T$ whose distance to $g_{c}$ is maximum is located. Both graphs $g_{c}$ and $g_{f}$ are selected as prototypes. The distance $d_{\max }=d\left(g_{c}, g_{f}\right)$ is then divided in $m-1$ partitions with interval $=\frac{d_{\max }}{m-1}$. The $m-2$ graphs that are located the nearest to the interval borders are selected as prototypes.

${ }^{1}$ For a detailed review of the applied prototype selection method we refer to 28 . 
Of course, one can imagine other techniques and strategies for prototype selection, for example, mixing some of the strategies described above with each other. The intention of all methods remains the same - finding a good selection of $m$ prototypes that lead to a good performance of the resulting classifier in the vector space.

\subsection{Dimensionality Reduction}

In Section 3.3 a number of prototype selectors have been introduced. Typically, these prototype selectors are tested on a validation set and the one that leads to the best classification performance is finally chosen. A similar procedure can be applied in order to find the optimal number of prototypes. That is, the number of prototypes is varied over a certain range and the number that results in the highest classification rate is adopted for the final system.

In the current section we describe an alternative approach where we use all available elements from the training set as prototypes, i.e. $P=T$ and subsequently apply dimensionality reduction methods. This process is more principled and allows us to completely avoid the problem of finding the optimal prototype selection strategy. For dimensionality reduction, we make use of the well known Principal Component Analysis (PCA) and Fisher's Linear Discriminant Analysis (LDA) 129.

The Principal Component Analysis (PCA) [129] is a linear transformation. It seeks the projection which best represents the data. PCA is an unsupervised method which does not take any class label information into consideration. We first normalize the data by shifting the mean to the origin of the coordinate system and making the variance of each feature equal to one. Then we calculate the covariance matrix of the normalized data and determine the eigenvectors $\mathbf{e}_{i}$ and the eigenvalues $\lambda_{i}$ of the covariance matrix. The eigenvectors are ordered according to decreasing magnitude of the corresponding eigenvalues, i.e. $\lambda_{1} \geq$ $\lambda_{2} \geq \ldots \geq \lambda_{n} \geq 0$. The data is then represented in a new coordinate system defined by the eigenvectors. For reducing the dimensionality of the transformed data we retain only the $m<n$ eigenvectors with the $m$ highest eigenvalues.

Fisher's Linear Discriminant Analysis (LDA) [129] is a linear transformation as well. In contrast with PCA, LDA takes class label information into account. In its original form, LDA can be applied to two-class problems only. However, we make use of a generalization, called Multiple Discriminant Analysis (MDA), which can cope with more than two classes. In MDA, we are seeking the projection of the data which best separates the classes from each other. For all further details, we refer to [129]. Note that under this transformation the maximal dimensionality of the transformed feature space is $c-1$, where $c$ is the number of classes.

\subsection{Multiple Classifier System}

Recently, the field of multiple classifier systems has become a very active area of research. The fundamental observation that motivates the combination of 
classifiers is that the sets of patterns misclassified by different classifiers do not necessarily heavily overlap. Hence, errors of a single classifier can be compensated by the other classifiers of an ensemble [30]. In the case of statistical patterns, that is, patterns represented by feature vectors, a large number of methods for the creation and combination of classifiers have been developed over the past years 313233 .

Regarding the family of graph kernels proposed in this paper, if we repeat the process of random prototype selection a number of times, we can derive different graph subsets that can be used to map a given population of graphs to various vector spaces. As a result, one can create a statistical classifier ensemble for structural input data.

In Algorithm 1 the random prototype selection strategy is described. This procedure selects $n$ times a subset of size $m$ out of the training set. Once a graph has been selected it becomes temporarily unavailable until all training patterns have been selected. This is achieved by a tabu list, which contains all patterns that are already selected (line 11). The randomized prototype selection is performed on the subset $T \backslash T A B U$ only (line 9). Whenever the tabu list contains all patterns of $T$, a reset is done such that all training elements become available again (line 6 and 7 ).

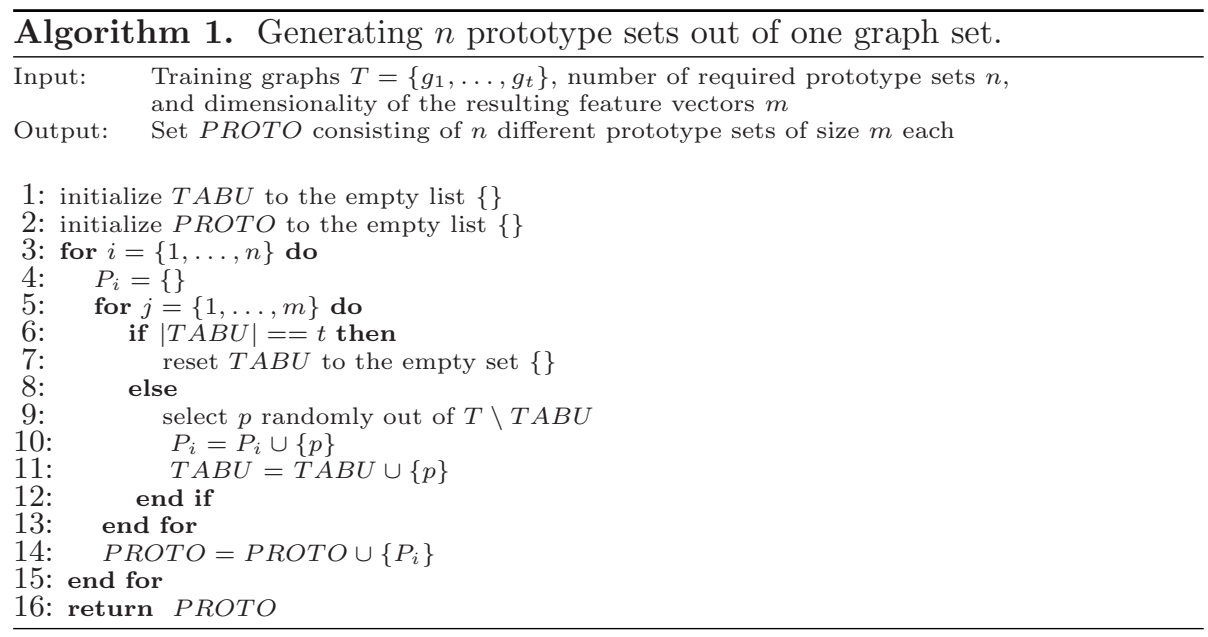

To generate a classifier ensemble, we apply a methodology which is known as overproduce-and-select 30. The idea is to produce a pool of classifiers, followed by a selection procedure that picks the classifiers that are most diverse and accurate. We make use of the accuracy of the resulting ensembles to control the selection of a subset out of the classifier pool. To create a good performing ensemble a sequential floating search selection 34 is applied. For the combination of the individual ensemble member outputs, plurality voting, Borda count and linear score combination have been applied, depending on the type of output delivered by the individual classifier. 


\section{Experimental Results}

In this section we provide the results of an experimental evaluation of the proposed embedding kernels. We aim at empirically confirming that the method of graph embedding and subsequent classification in real vector spaces is applicable to different graph classification problems and matches, or even surpasses, the performance of a $k$-nearest neighbor classifier $(k-\mathrm{NN})$ in the original graph domain. Note that $k$-NN classifiers are the only classifiers that can be directly applied in the original graph domain. The classifier used in the vector space is the SVM [5]. Of course, any other classifier could be used for this purpose as well. However, we feel that the SVM is particularly suitable because of its theoretical advantages and its superior performance that has been empirically confirmed in many practical classification problems.

For further results achieved with the novel family of graph kernels, especially for comparisons with other reference systems, we refer to [7/8928.

\subsection{Experimental Setup}

In each of our experiments we make use of three disjoint graph sets, viz. validation set, test set and training set. The validation set is used to determine optimal parameter values for graph embedding, ensemble generation, and classification. The embedding parameters consist of the number of prototypes, i.e. the dimensionality of the resulting vector space, and the best performing embedding method ${ }^{2}$. The parameters for ensemble generation consist of the optimal dimensionality of the random prototype sets and the optimal members of the classifier ensemble. Finally, the parameters for classification consist of parameter $k$ for the nearest neighbor classifier and the different parameters for the SVM. The RBF-kernel SVM used in this paper has parameters $C$ and $\gamma$, where $C$ corresponds to the weighting factor for misclassification penalty and $\gamma$ is used in our kernel function $K(\mathbf{u}, \mathbf{v})=\exp \left(-\gamma \cdot\|\mathbf{u}-\mathbf{v}\|^{2}\right)$. The parameter values, the embedding method, the classifier ensemble, and the dimensionality that result in the lowest classification error on the validation set are then applied to the independent test set.

\subsection{Databases}

For our experimental evaluation, four data sets with quite different characteristics are used. The datasets vary with respect to graph size, edge density, type of labels for the nodes and edges, and meaning of the underlying objects.

The first database used in the experiments consists of graphs representing distorted letter drawings. In this experiment we consider the 15 capital letters of the Roman alphabet that consist of straight lines only $(A, E, F, \ldots)$. For each class, a prototype line drawing is manually constructed. To obtain aribtrarily large sample sets of drawings with arbitrarily strong distortions, distortion operators are applied to the prototype line drawings. This results in randomly translated, removed,

\footnotetext{
${ }^{2}$ Note that with dimensionality reduction algorithms (PCA and LDA) only the dimensionality has to be validated.
} 
and added lines. These drawings are then converted into graphs by representing lines by edges and ending points of lines by nodes. Each node is labelled with a twodimensional attribute giving its position. The graph database used in our experiments is composed of a training set, a validation set, and a test set, each of size 750 .

For a more thorough evaluation of the proposed methods we additonally use three real world data sets. First we apply the proposed method to the problem of image classification. Images are converted into graphs by segmenting them into regions, eliminating regions that are irrelevant for classification, and representing the remaining regions by nodes and the adjacency of regions by edges [35]. The image database consists of five classes (city, countryside, people, snowy, streets) and is split into a training set, a validation set and a test set of size 54 each.

The second real world dataset is given by the NIST-4 fingerprint database 36. We construct graphs from fingerprint images by extracting characteristic regions in fingerprints and converting the results into attributed graphs [37]. We use a validation set of size 300 and a test and training set of size 500 each. In this experiment we address the 4-class problem (arch, left-loop, right-loop, whorl).

Finally, we apply our novel graph kernels to the problem of molecule classification. To this end, we construct graphs from the AIDS Antiviral Screen Database of Active Compounds 38. Our molecule database consists of two classes (active, inactive), which represent molecules with activity against HIV or not. We use a validation set of size 250, a test set of size 1500 and training set of size 250 . Thus, there are 2000 elements in total (1600 inactive elements and 400 active elements). The molecules are converted into graphs by representing atoms as nodes and the covalent bonds as edges. Nodes are labelled with the number of the corresponding chemical symbol and edges by the valence of the linkage.

\subsection{Results and Discussion}

In Table 1 we provide the classification accuracy on all described datasets achieved with all of the proposed methods. In the first row of the embedding kernel classifiers the classification results achieved with an SVM based on prototype selection and graph embedding are given. Note that this kernel outperforms the reference system on all datasets, in four out of six cases with statistical significance. Similar results can be observed with the SVM based on the PCA reduced vectors where three out of six improvements are statistically significant. The SVM based on LDA reduced vectors perform poorly on the fingerprint and molecule data. Note that with LDA reduction the maximal dimensionality of the transformed feature space is $c-1$, where $c$ is the number of classes, i.e. on the fingerprint graphs three dimensions are used and on the molecule graphs only one dimension is used for classification. Nevertheless, on the remaining databases three out of four improvements over the reference system are statistically significant. The last column of Table 1 provides results achieved with a multiple classifier system based on plurality voting. Compared to the reference system in the graph domain four out of six improvements are statistically significant. Note that this system achieves three times the overall best result among all proposed methods (Letter high, Fingerprints, and Molecules). 
Table 1. Classification accuracy in the original graph domain and in the embedded vector space

\begin{tabular}{|c|c|c|c|c|c|}
\hline \multirow[b]{2}{*}{ Database } & \multirow{2}{*}{$\frac{\text { Ref. System }}{k \text {-NN (graph) }}$} & \multicolumn{4}{|c|}{ Embedding Kernel Classifiers } \\
\hline & & Prototype-SVM & PCA-SVM & LDA-SVM & Plurality Voting \\
\hline Letter (low) & 98.3 & 98.5 & 98.5 & 99.1 & 98.3 \\
\hline Letter (med) & 94.0 & $96.9 \circ$ & $97.2 \circ$ & $96.5 \circ$ & $97.1 \circ$ \\
\hline Letter (high) & 90.1 & $92.9 \circ$ & $93.7 \circ$ & $94.0 \circ$ & $94.3 \circ$ \\
\hline Image & 57.4 & 64.8 & 61.1 & $68.5 \circ$ & 61.1 \\
\hline NIST-4 & 82.6 & $85.0 \circ$ & 84.6 & $66.6 \bullet$ & $85.2 \circ$ \\
\hline Molecules & 97.1 & $98.1 \circ$ & $98.2 \circ$ & $95.3 \bullet$ & $98.3 \circ$ \\
\hline
\end{tabular}

\section{Conclusions}

Although graphs have a higher representational power than feature vectors, there is a lack of methods for pattern classification using graph representations. By contrast, a large number of methods for classification have been proposed for object representations given in terms of feature vectors. The present paper introduces a novel family of graph kernels in order to bridge the gap between structural and statistical pattern recognition. These graph kernels make explicit use of graph edit distance and can therefore deal with various kinds of graphs (labelled, unlabelled, directed, undirected, etc.). The basic idea of the embedding kernel is to describe a graph by means of $m$ dissimilarities to a predefined set of graphs termed prototypes. That is, a graph $g$ is mapped explicitly to the $m$-dimensional real space $\mathbb{R}^{m}$ by arranging the edit distances of $g$ to all of the $m$ prototypes as a vector. We show that the embedding process can be controlled by different prototype selectors or by well-known dimensionality reduction algorithms. By means of this procedure one obtains not only pairwise scalar products in an implicit kernel feature space but also the maps of the individual graphs. Furthermore, the proposed family of graph kernels lends itself to a method for the automatic generation of classifier ensembles. From the results of our experiments, one can conclude that the classification accuracy can be statistically significantly enhanced by all embedding methods but LDA on all considered databases.

\section{Acknowledgements}

This work has been supported by the Swiss National Science Foundation (Project 200021-113198/1).

\section{References}

1. Duda, R., Hart, P., Stork, D.: Pattern Classification, 2nd edn. Wiley-Interscience, Chichester (2000)

2. Schölkopf, B., Smola, A.: Learning with Kernels. MIT Press, Cambridge (2002) 
3. Shawe-Taylor, J., Cristianini, N.: Kernel Methods for Pattern Analysis. Cambridge University Press, Cambridge (2004)

4. Vapnik, V., Chervonenkis, A.: On the uniform convergence of relative frequencies of events to their probabilities. Theory of Probability and its Applications 16(2), 264-280 (1971)

5. Vapnik, V.: Statistical Learning Theory. John Wiley, Chichester (1998)

6. Gärtner, T.: A survey of kernels for structured data. SIGKDD Explorations 5(1), 49-58 (2003)

7. Riesen, K., Neuhaus, M., Bunke, H.: Graph embedding in vector spaces by means of prototype selection. In: Accepted for the 6th Int. Workshop on Graph-Based Representations in Pattern Recognition (2007)

8. Riesen, K., Kilchherr, V., Bunke, H.: Reducing the dimensionality of vector space embeddings of graphs. In: Accepted for the International Conference on Machine Learning and Data Mining MLDM (2007)

9. Riesen, K., Bunke, H.: Classifier ensembles for vector space embedding of graphs. In: Accepted for the 7th Int. Workshop on Multiple Classifier Systems (2007)

10. Gärtner, T., Flach, P., Wrobel, S.: On graph kernels: Hardness results and efficient alternatives. In: Schölkopf, B., Warmuth, M., eds.: Proc. 16th Annual Conf. on Learning Theory, pp. 129-143 (2003)

11. Borgwardt, K., Ong, C., Schönauer, S., Vishwanathan, S., Smola, A., Kriegel, H.P.: Protein function prediction via graph kernels. Bioinformatics 21(1), 47-56 (2005)

12. Mahé, P., Ueda, N., Akutsu, T.: Graph kernels for molecular structures - activity relationship analysis with support vector machines. Journal of Chemical Information and Modeling 45(4), 939-951 (2005)

13. Lafferty, J., Lebanon, G.: Diffusion kernels on statistical manifolds. Journal of Machine Learning Research 6, 129-163 (2005)

14. Haussler, D.: Convolution kernels on discrete structures. Technical Report UCSCCRL-99-10, University of California, Santa Cruz (1999)

15. Watkins, C.: Kernels from matching operations. Technical Report CSD-TR-98-07, Royal Holloway College (1999)

16. Jain, B., Geibel, P., Wysotzki, F.: SVM learning with the Schur-Hadamard inner product for graphs. Neurocomputing 64, 93-105 (2005)

17. Ramon, J., Gärtner, T.: Expressivity versus efficiency of graph kernels. In: Proc. First International Workshop on Mining Graphs, Trees and Sequences, 65-74 (2003)

18. Horvath, T., Gärtner, T., Wrobel, S.: Cyclic pattern kernels for predictive graph mining. In: Proc. International Conference on Knowledge Discovery and Data Mining, pp. 65-74. ACM Press, New York (2004)

19. Neuhaus, M.: Bridging the Gap Between Graph Edit Distance and Kernel Machines. World Scientific (to appear, 2007)

20. Bunke, H., Allermann, G.: Inexact graph matching for structural pattern recognition. Pattern Recognition Letters 1, 245-253 (1983)

21. Sanfeliu, A., Fu, K.: A distance measure between attributed relational graphs for pattern recognition. IEEE Transactions on Systems, Man, and Cybernetics (Part B) 13(3), 353-363 (1983)

22. Riesen, K., Neuhaus, M., Bunke, H.: Bipartite graph matching for computing the edit distance of graphs. In: Accepted for the 6th Int. Workshop on Graph-Based Representations in Pattern Recognition (2007)

23. Neuhaus, M., Riesen, K., Bunke, H.: Fast suboptimal algorithms for the computation of graph edit distance. In: Ribeiro, C.C., Martins, S.L. (eds.) WEA 2004. LNCS, vol. 3059, pp. 163-172. Springer, Heidelberg (2004) 
24. Wilson, R., Hancock, E., Luo, B.: Pattern vectors from algebraic graph theory. IEEE Trans. on Pattern Analysis ans Machine Intelligence 27(7), 1112-1124 (2005)

25. Wilson, R., Hancock, E.: Levenshtein distance for graph spectral features. In: Proc. 17th Int. Conf. on Pattern Recognition, vol. 2, pp. 489-492 (2004)

26. Luo, B., Wilson, R., Hancock, E.: Spectral embedding of graphs. Pattern Recognition 36(10), 2213-2223 (2003)

27. Duin, R., Pekalska, E.: The Dissimilarity Representations for Pattern Recognition: Foundations and Applications. World Scientific (2005)

28. Riesen, K., Bunke, H.: A graph kernel based on vector space embedding (Submitted)

29. Bishop, C.: Neural Networks for Pattern Recognition. Oxford University Press, Oxford (1996)

30. Kuncheva, L.: Combining Pattern Classifiers: Methods and Algorithms. John Wiley, Chichester (2004)

31. Breiman, L.: Bagging predictors. Machine Learning 24, 123-140 (1996)

32. Ho, T.: The random subspace method for constructing decision forests. IEEE Trans. on Pattern Analysis ans Machine Intelligence 20(8), 832-844 (1998)

33. Freund, Y., Shapire, R.: A decision theoretic generalization of online learning and application to boosting. Journal of Computer and Systems Sciences 55, 119-139 (1997)

34. Pudil, P., Novovicova, J., Kittler, J.: Floating search methods in feature-selection. Pattern Recognition Letters 15(11), 1119-1125 (1994)

35. Le Saux, B., Bunke, H.: Feature selection for graph-based image classifiers. In: Marques, J.S., Pérez de la Blanca, N., Pina, P. (eds.) IbPRIA 2005. LNCS, vol. 3523, pp. 147-154. Springer, Heidelberg (2005)

36. Watson, C., Wilson, C.: NIST special database 4, fingerprint database. National Institute of Standards and Technology (1992)

37. Neuhaus, M., Bunke, H.: A graph matching based approach to fingerprint classification using directional variance. In: Kanade, T., Jain, A., Ratha, N.K. (eds.) AVBPA 2005. LNCS, vol. 3546, pp. 191-200. Springer, Heidelberg (2005)

38. DTP, D.T.P.: Aids antiviral screen (2004) http://dtp.nci.nih.gov/docs/aids/ aids_data.html 